\title{
On the Design and Implementation of Efficient Antennas for HF RFID Readers
}

\author{
Ernest Ofosu Addo ${ }^{1}$, Benjamin Kommey ${ }^{2}$, Andrew Selasi Agbemenu ${ }^{3}$, and Hermann \\ Kumbong $^{3}$ \\ ${ }^{1}$ University of Siena \\ ${ }^{2}$ Kwame Nkrumah University of Science and Technology College of Arts and Social Science \\ ${ }^{3}$ Kwame Nkrumah University of Science and Technology
}

August 18, 2020

\begin{abstract}
This paper describes an in-depth methodical approach to the development of efficient high frequency (HF) antennas for usage in radio frequency identification (RFID) systems operating at $13.56 \mathrm{MHz}$. It presents brief theory relevant to RFID communication and sets up a framework within which features and requirements of antennas are linked to key design parameters such as antenna form-factor and size; RF power level, materials and communication protocol. Tuning circuits necessary to adjust the resonance and power matching characteristics of antennas for good transponder interrogation and response recovery are discussed. To validate the approaches outlined, a complete step-wise antenna design and measurement described. Common practical problems that are often encountered in such design processes are also commented on.
\end{abstract}

\section{Hosted file}

wileyNJD-Doc.pdf available at https://authorea.com/users/351992/articles/476390-on-thedesign-and-implementation-of-efficient-antennas-for-hf-rfid-readers 